\title{
Automatic Segmentation of Connected Thin Peripheral Vessels in Chest CT Scan Images
}

\author{
P. Ammi Reddy \\ Vasireddy Venkatadri Institute of \\ Technology \\ Nambur, Guntur \\ Andhra Pradesh, India
}

\author{
E. V. Krishna Rao \\ Andhra Loyola Institute of \\ Engineering \& Technology \\ Vijayawada \\ Andhra Pradesh, India
}

\author{
I. Ramesh Babu \\ Acharya Nagrjuna University \\ Nagarjuna Nagar \\ Guntur \\ Andhra Pradesh, India
}

\begin{abstract}
Vessel enhancement and segmentation is one of the crucial pre-processing steps in accurate vessel tree reconstruction in many chest CT scan imaging applications. Conservative vessel enhancement approaches used eigenvalues of Hessianbased filters, which are found to be sensitive to noise, fails to detect small ones, and sometimes give discontinued vessels due to junction suppression. Since Hessian-based filters cannot distinguish step edges from vessels effectively, in this paper, we propose a novel framework to overcome the problems for vessel enhancement for thorax CT images. The road map of proposed work has three steps. First, extract the lung region from thorax CT images based on Gray-level thresholding and morphological operations; then, according to the idea of the matched filter in different directions, and local entropy thresholding, to obtain more precise analysis in noisy environment and thus can correctly reveal entire vessels. Also, qualitative as well as quantitative evaluations performed on CT images show that the proposed filter generates better performance in comparison against two Hessian-based approaches (Frangi and Shikata)..
\end{abstract}

\section{Keywords}

Vessel enhancement, Hessian-based, matched filter, computer aided detection.

\section{INTRODUCTION}

In recent times due to the development of multi-slice computer tomography (CT) technology, a modern CT scanner can generate a large number (200-1000) of slices for each patient, which can cover a large volume of human body within the short time. Using this high performance technology, radiologists can easily photograph the whole human chest, abdomen with high spatial resolution in a onetime CT scan. Observation of complicated anatomical structures in the human body and discovery of small abnormal regions in a different organ has become possible with this technology.

Two basic functions viz., abnormality detection and visualization of CT images are necessary to develop a computer aided diagnosis system (CAD) that helps automatic detection of anatomical structures. They are pre-segmentation of the principal human organ regions and recognition of human structures from CT images. In the case of chest CT imaging, the lung is the principal region. Lung structure is constructed by lung vessels, bronchus, and lung fissures. These are the important elements which play a vital role in diagnosing the lung cancer, pneumonia and diffuse lung diseases decisions in clinical diagnosis. Identifying the lung structure is the most basic and indispensable aspect of successful CAD, and undoubtedly influences the efficiency of the overall CAD system [1].
The automatic segmentation of the pulmonary vasculature is an essential pre-processing step for many algorithms that analyze thoracic CT scans. An accurate segmentation can be used to improve the nodule detection by excluding the vessels from lung region. The vessel tree segmentation which is generated by the pulmonary vasculature also can be used to detect pulmonary embolisms and for quantification of hypertension in which the diameter of the arteries provide an indicator for cardiovascular risk. Furthermore, segmentation of the pulmonary vascular tree can be used as a first step for other algorithms such as artery vein separation algorithms.

The pulmonary vascular tree is a complex and heavily intertwined tree structure, with many generations for the arterial and for the venous tree and diameters varying from approximately $20 \mu \mathrm{m}-15 \mathrm{~mm}$ [2]. Blood has an intensity of around 40 Hounsfield Units (HU) on CT images. However, due to the partial volume effect the maximum intensity of small vessels is significantly lower than that of large, which makes intensity based global thresholding difficult. Another problem caused by partial volume effects is that vessels in close proximity to each other are hard to discriminate as separate entities. It is very difficult to differentiate between vessel crossings and bifurcations.

Segmentation of Pulmonary vessel tree plays a pivotal role in the diagnosis of vascular diseases such as stenosis, hypertension, and pulmonary embolism. The vessel structure is also an important cue for registering images of the same patient [3]. Particularly in clinical practice, it is of great importance to be able to characterize the vascular trees for the detection of pulmonary emboli, detection of signs of pulmonary hypertension, and for the differentiation between vasculature and focal opacitie. The vascular trees can also serve as a roadmap for monitoring the lung tissues as in the aspect of lung volume changes and time taken for that change. The extraction of vessel tree aids to improve the detection of pathological structures like lung nodules where it has been shown to help in reducing false positives [4].

The road map of the proposed work has three stages. During the first stage, the lung region is extracted from chest CT images. The second stage involves vessel enhancement based on the matched filters, and finally local entropy thresholding is estimated. Section 2 describes literature survey. Proposed algorithm has been explained in the section 3. Section 4 consists of the results and performance evaluation by the existing methods, and finally discussion followed by conclusion in section 5 .

\section{LITERATURE REVIEW}

Many vessel enhancing filters have been proposed by many researchers based on second order derivatives. These filters use a priori information about the principle directions of vessels, which are expressed in terms of eigenvalues of the 
Hessian matrix. Different response functions have been proposed based on different combinations of these eigenvalues to enhance vessels and suppress other bright structures, such as nodules, junctions, fissures, airway walls and noise [3-8]. The first order derivatives have also been used for vessel enhancement, but they claim that these are less sensitive to noise. To segment the vessel tree, the output of the vessel enhancement filters may be thresholded. However, the filter response can also be used as input for a vessel reconstruction algorithm, for instance based on mathematical morphology [4].

Different classes of vessel segmentation algorithms use region grow based algorithms such as fast marching level-set theory [9] and wave front propagation [10]. Starting from a seed the vessel tree is traced by iteratively expanding a front to a given speed function consisting of criteria that determine whether the voxels under consideration are part of a vessel or not. Resulting in a connected vessel tree detection and surface reconstruction are performed simultaneously within this class of algorithms. Certain other tracking algorithms or optimal path algorithms have also been used for vessel segmentation. Cost function from a multiscale vessel enhancement filter [11] is such an example. An active contour model is also proposed for vessel tree segmentation [12]. To segment thick blood vessels, a region competition-based active contour model which uses the Gaussian mixture model is applied. A vector field derived from the eigen-analysis of the Hessian matrix in a multiscale framework is used to segment thin blood vessels.

Although, most of these algorithms take shape features into account, effective suppression of noise and high density structures such as airway walls, fissures, and abnormalities remains an important issue in this regard. This is also the case because many algorithms have been designed to detect tubular structures in general and have been applied to coronary arteries, vessels in the brain and even for bone structures in particular. Since the lungs contain many bright structures, of which sonic have similar characteristics as vessels, these algorithms might not be capable of differentiating. The two Hessian-based approaches, Frangi and Shikata filters were reviewed since both are considered as standard techniques in the following lines.

\subsection{Frangi Filter}

The Frangi Filter is based on the eigenvalues of the Hessian matrix computed at selected locations and at multiple scales. The multiple scales are obtained by smoothing the data with different size Gaussian kernels.

Let $\left|\lambda_{1}\right| \leq\left|\lambda_{2}\right|$ be the eigenvalues of the Hessian matrix and let $\mathrm{e}_{1}, \mathrm{e}_{2}$ be the eigenvector associated with $\lambda_{1}, \lambda_{2}$ respectively. Based on the vessel assumptions above the ratio $\left|\lambda_{2}\right| /\left|\lambda_{1}\right|$ should be close to zero and $\mathrm{e}_{1}$ should be in the cross section of the vessel whereas $e_{2}$ should be in the direction of the center line. Based on these assumptions, Frangi proposed the following vessel enhancement filter:

$$
\psi_{\mathrm{S}}(\mathrm{x})=\eta\left(\lambda_{1}\right)\left(\exp \left(-\frac{\mathbf{R}_{\mathrm{b}}^{2}}{2 \beta^{2}}\right) \exp \left(-\frac{\mathbf{R}_{\mathrm{c}}^{2}}{2 \gamma^{2}}\right)\right)
$$

where $\mathrm{S}$ is the scale of the filter, $\mathrm{x}$ is the location of the filter. $B$ and $\gamma$ are constant normalization factors $R_{b}$ and $R_{c}$ are given based on the eigenvalues of the Hessian matrix:

$$
\begin{gathered}
R_{b}=\frac{\left|\lambda_{2}\right|}{\left(\left|\lambda_{1}\right|\right)^{2}} \\
R_{c}=\left(\lambda_{1}^{2}+\lambda_{2}^{2}\right)^{2}
\end{gathered}
$$

The function $\eta\left(\lambda_{1}\right)$ is set to 1 when $\lambda_{1}<0$ and set to 0 otherwise. Given a set of several scales $\mathrm{T}$, the filter output at location $x$ is given by

$$
\Psi(\mathrm{x})=\max \left\{\Psi_{\mathrm{S}}(\mathrm{x}) \mid \mathrm{S} \varepsilon \mathrm{T}\right\}
$$

\subsection{Shikata Filter}

Based on almost similar considerations and the eigenvalue computation of Frangi, Shikata defines a vessel enhancement filter at a single scale as

$$
\psi_{\mathrm{S}}(\mathrm{x})=\max _{\mathrm{S} \varepsilon \mathrm{T}} \eta\left(\lambda_{1}\right)\left(\frac{\mathrm{S}^{2} \lambda_{2}}{\mathrm{I}(\mathrm{x})}\right)
$$

where $\mathrm{I}(\mathrm{X})$ is the pixel intensity at location $\mathrm{x}$.

\section{PROPOSED ALGORITHM}

The flowchart of our integrated algorithm in Fig 1 consists of three main procedures to segment the pulmonary blood vessels from the input chest CT images: (1) a mask is used to exclude objects which are not part of a vessel in order to limit computation time i.e. lung segmentation using conventional methods, (2) vessel enhancement based on the different directional matched filters, (3) local entropy threshold to remove grains occurred during enhancement. The details of each step are introduced below.

\subsection{Lung segmentation}

Image denoising and histogram enhancement techniques were used to improve image quality and enhance the contrast between different objects. Gray-level thresholding is used to extract lung regions from the original CT image. The algorithm assumes that the image contains two classes of pixels (background and foreground) and can find an optimal threshold by iterative calculations through all possible values. The aim of Otsu's method [13] is to find the optimum threshold value to separate the two classes where their combined spread (intra-class variance) is nominal.

The gray level image is converted to a binary image after the calculation of an optimum threshold. The lung parenchyma is then extracted from the segmented thoracic region by applying a flood fill operator that starts from the four corners of the thresholded image. The black lung region of the lung parenchyma contains small white shapes representing nodules and blood vessels. To eliminate them, the white shapes are labeled using connected component labeling. After all white shapes in the lung region are labeled; their sizes are analyzed using the following rule. 


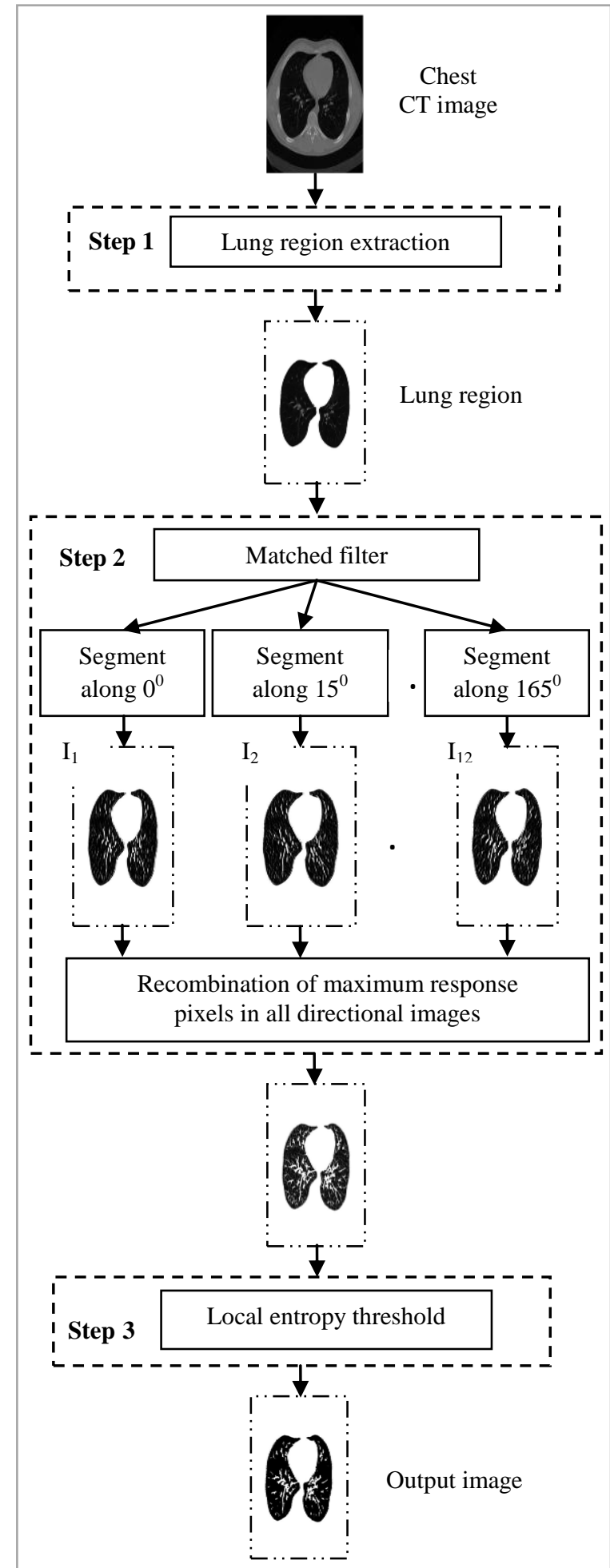

Fig 1: Flowchart of the proposed enhancement frame work. There are three main steps: Step1: extraction of lung region from chest $\mathrm{CT}$ images, Step2: vessel enhancement using matched filters and Step3: local entropy thresholding.

$\mathrm{I}(\mathrm{x}, \mathrm{y})=1$, if $\mathrm{S}(\mathrm{k})$ is smaller than 150 pixels, where $\mathrm{k}$ is the label of the white shape

The resulted mask image is subtracted from original image to get the intact lung region. The results are shown in Fig 2.

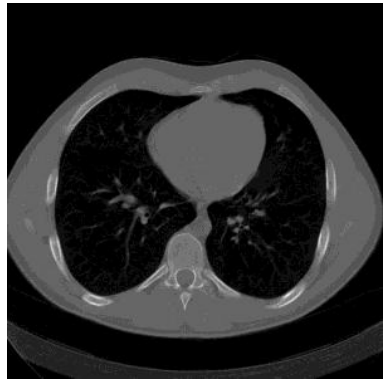

(a)

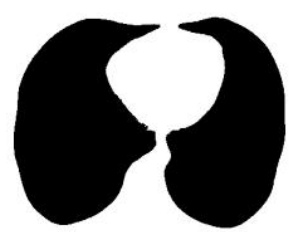

(c)

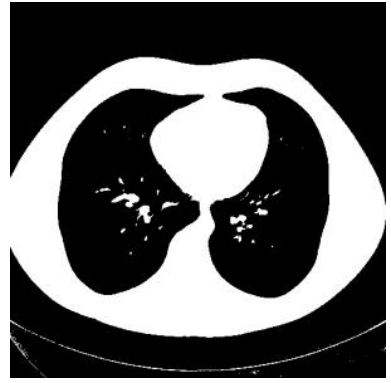

(b)

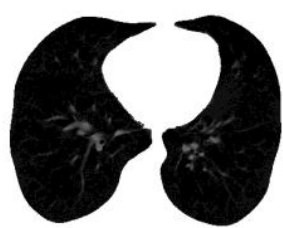

(d)
Fig 2: Results for chest CT image. (a) Original CT image, (b) by segmentation of the original image using Otsu's thresholding method, (c) by mask after application of morphological operations, and (d) by final intact lung parenchyma extraction.

\subsection{Matched Filter}

The intensity profile of the cross section of blood vessels can be approximated by a Gaussian shaped curve, the intensity changes little along the center line of vessels, and there are two edges at the boundary of vessels. We assume that the vessels in lung region have following three properties:

1) The blood vessels may be approximated as piecewise linear segments as they have small curvatures.

2) The lung vessel has much higher density than the background of lung region. A few representative samples of blood vessel intensity profiles along directions perpendicular to their length are plotted in Fig.3. It was observed that these vessels almost never have ideal step edges. Even though the intensity profile varies by a small amount from vessel to vessel, it may be approximated by a Gaussian curve:

$$
f(x, y)=k \exp \left(-\frac{d^{2}}{2 \sigma^{2}}\right)
$$

where $d$ is the perpendicular distance between the point (x, y) and the straight line passing through the center of the blood vessel in a direction along its length, $\sigma$ is the spread of the intensity profile, and $\mathrm{k}$ indicates the measure of reflectance of the vessel relative to its neighborhood.

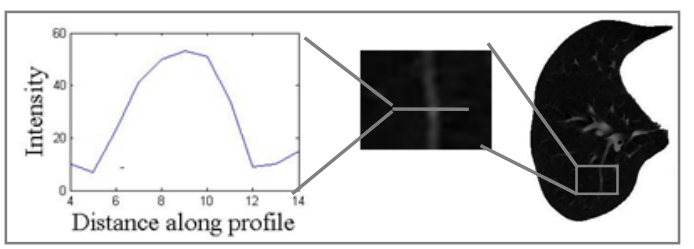

Fig 3: The gray level profiles of the cross section of blood vessels in the left lung lobe.

3) The width of a blood vessel decreases as it goes radially outward from the hilum, such a change in vessel caliber is a gradual one. The diameters of the vessels are found to lie 
within that range of approximately $20 \mu \mathrm{m}-15 \mathrm{~mm}$. For our initial calculations, however, we shall assume that all the blood vessels in the image are of equal width $3 \sigma$.

Therefore, a blood vessel is defined as a bright pattern having Gaussian shape cross-section profile, piecewise connected, and locally linear.

Now, let us consider the concept of matched filter detection [14] used to detect blood vessels in the segmented lung regions. The two-dimensional matched filter kernel is designed to convolve with the original segmented lung image in order to enhance the blood vessels. A prototype matched filter kernel is expressed as

$$
f(x, y)=\exp \left(-\frac{x^{2}}{2 \sigma^{2}}\right) \text { for }|y| \leq \frac{L}{2}
$$

where $L$ indicates the length of blood vessel segment for which the blood vessel is having a fixed orientation. The direction of the vessel is considered to be aligned along the $y-$ axis. Since a blood vessel may be oriented at any angle $\theta(0 \leq$ $\theta \leq \pi)$, the kernel needs to be rotated for all possible angles. The matched filter $\mathrm{f}(\mathrm{x}, \mathrm{y})$ will have its maximum response only when it is aligned at an angle $\theta \pm \pi / 2$. Thus, the matched filter kernel is needs to be rotated for all possible angles since a blood vessel may be oriented at any angle.

In [8], twelve different kernels have been constructed to span all possible orientations. A set of twelve $15 \times 15$ pixel kernels are applied by convolving to a segmented lung image and at each pixel only the maximum of their responses is retained. The results after convolving matched filter kernels at different angles with lung region of the chest CT image in Fig 2 (d) are shown in Fig 4(a-l) and the maximum of their responses is retained as shown in Fig 4(m) where the blood vessels are significantly enhanced. During this process the contrast of the background tissues are also enhanced along with blood vessels.

\subsection{Local Entropy Thresholding}

In order to properly segment the enhanced blood vessel segments in the matched filter response (MFR) images, an effective thresholding scheme is need. As some of the MFR images have complicated relationships or overlap between foreground and background, efficient local entropy based thresholding algorithm that takes into account the spatial distribution of gray levels is used. Particularly, we implement a local entropy thresholding technique, described in [15] which can well maintain the structure details in the thresholded image.

Two images with identical histograms but different spatial distribution will result in different entropy and also different threshold values. The co-occurrence matrix of an image is a $P$ $\mathrm{x}$ Q dimensional matrix, represented by $\mathrm{T}=\left[\mathrm{t}_{\mathrm{ij}}\right]_{\mathrm{PXQ}}$ that gives an idea about the transition of grey level between two adjacent pixels, indicating spatial structural information of an image. More specifically, let $t_{i j}$ be the $(i, j)^{\text {th }}$ element of the cooccurrence matrix $\mathrm{T}$, different definitions of co-occurrence matrix are possible.

Here, we made the co-occurrence matrix asymmetric by considering the horizontally right and vertically lower transitions. Thus, $t_{i j}$ is defined as follows:

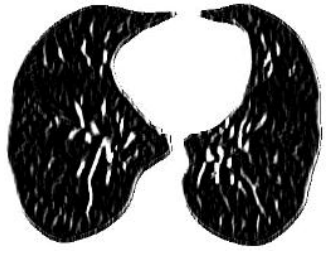

(a) $0^{0}$

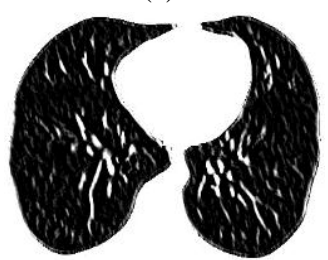

(c) $30^{\circ}$

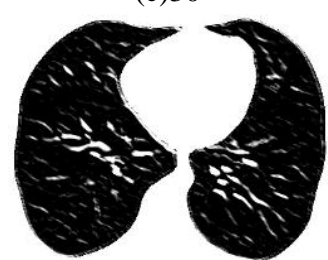

(e) $60^{\circ}$

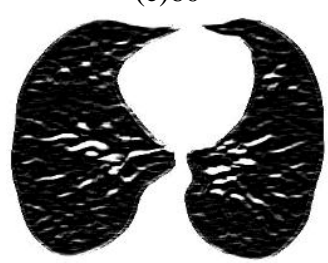

(g) $90^{0}$

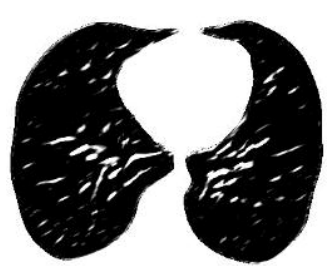

(i) $120^{0}$

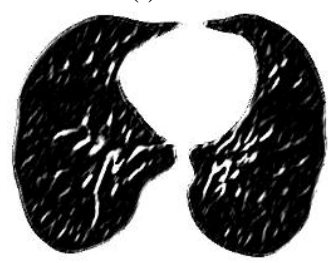

(k) $150^{0}$

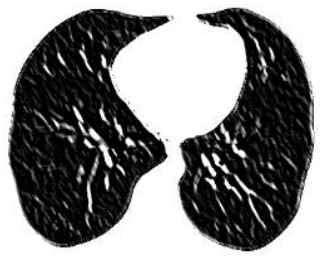

(b) $15^{0}$

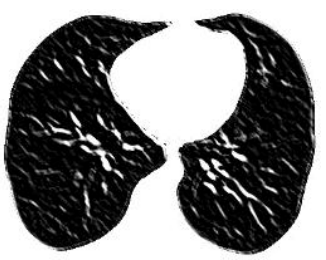

(d) $45^{0}$

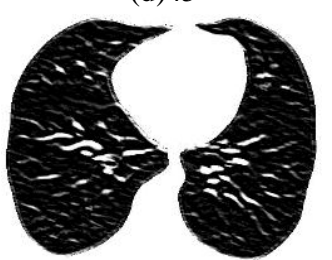

(f) $75^{0}$

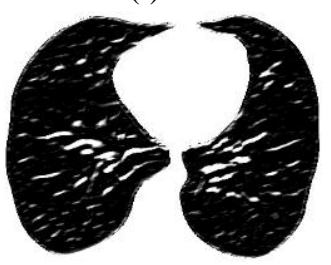

(h) $105^{0}$

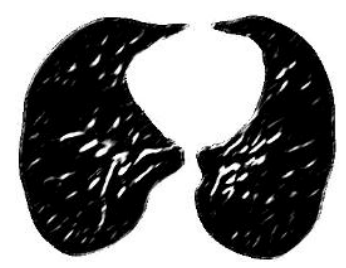

(j) $135^{0}$

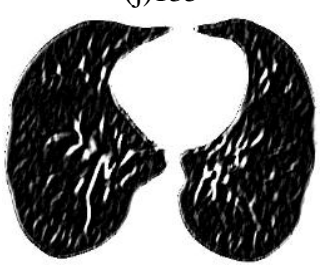

(1) $165^{0}$

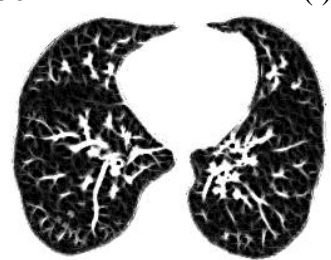

(m)Output image

Fig 4: Twelve-directional images of the segmented CT image shown in Fig 2(d) after applying matched filter and the maximum of their responses is retained in output image. 


$$
\begin{aligned}
& \mathrm{t}_{\mathrm{ij}}=\sum_{\mathrm{l}=1}^{\mathrm{P}} \sum_{\mathrm{k}=1}^{\mathrm{Q}} \delta_{\mathrm{lk}} \\
& \delta_{\mathrm{lk}}=\left\{\begin{array}{l}
1 \text { if }\left\{\begin{array}{l}
\mathrm{f}(1, \mathrm{k})=\mathrm{i} \text { and } \mathrm{f}(1, \mathrm{k}+1)=1 \text { and } / \text { or } \\
\mathrm{f}(1, \mathrm{k})=\mathrm{i} \text { and } \mathrm{f}(1+1, \mathrm{k})=1
\end{array}\right. \\
0 \text { otherwise }
\end{array}\right.
\end{aligned}
$$

where 'and/or' used in the $\delta_{\mathrm{lk}}$ earlier implies 'either or both'

Normalizing the total number of transitions in the cooccurrence matrix, a desired transition probability from grey level $\mathrm{i}$ to grey level $\mathrm{j}$ is obtained by

$$
\mathbf{p}_{\mathrm{ij}}=\frac{\mathbf{t}_{\mathrm{ij}}}{\sum_{\mathrm{l}=0}^{\mathrm{L}-1} \sum_{\mathrm{k}=0}^{\mathrm{L}-1} \mathbf{t}_{\mathrm{lk}}}
$$

If $\mathrm{s}, 0 \leq \mathrm{s} \leq \mathrm{L}-1$ is a threshold $\mathrm{t}$. Then $\mathrm{s}$ can partition the cooccurrence matrix into four quadrants, namely A, B, C, and D (see Fig 5). Here, it is assumed that pixels having gray level above the threshold value are assigned to foreground (corresponding to objects). Pixels having gray level equal to or below the threshold value are allocated to the background.

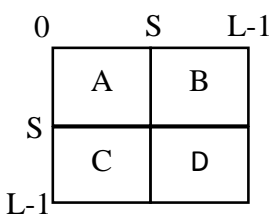

\section{Fig 5: Four quadrants of a co-occurrence matrix}

Let us define the following quantities:

$$
\begin{aligned}
& \mathbf{P}_{\mathrm{A}}=\sum_{\mathrm{i}=0}^{\mathrm{S}} \sum_{\mathrm{j}=0}^{\mathrm{S}} \mathrm{p}_{\mathrm{ij}} \\
& \mathbf{P}_{\mathrm{C}}=\sum_{\mathrm{i}=\mathrm{S}+1}^{\mathrm{L}-1} \sum_{\mathrm{j}=\mathrm{S}+1}^{\mathrm{L}-1} \mathrm{p}_{\mathrm{ij}}
\end{aligned}
$$

Normalizing the probabilities in each quadrant, such that the sum of the probabilities of each quadrant equals one, we get the following cell probabilities for different quadrants:

$$
P_{i j}^{A}=\frac{p_{i j}}{P_{A}} \text { for } O \leq i \leq s, O \leq j \leq s
$$

Similarly,

$$
\mathrm{P}_{\mathrm{ij}}^{\mathrm{C}}=\frac{\mathrm{P}_{\mathrm{ij}}}{\mathrm{P}_{\mathrm{C}}} \text { for } \mathrm{S}+1 \leq \mathrm{i} \leq \mathrm{L}-1, \mathrm{~S}+1 \leq \mathrm{j} \leq \mathrm{L}-1
$$

The second order entropy of the object can be defined as

$$
\mathbf{H}_{\mathrm{A}}^{(2)}(\mathrm{S})=-\frac{1}{2} \sum_{\mathrm{i}=0}^{\mathrm{S}} \sum_{\mathrm{j}=0}^{\mathrm{S}} \mathbf{P}_{\mathrm{i} J}^{\mathrm{A}} \log _{2}^{\mathrm{P}_{\mathrm{i}}^{\mathrm{A}}}
$$

Similarly, the second-order entropy of the background can be written as

$$
H_{C}^{(2)}(S)=-\frac{1}{2} \sum_{i=S+1}^{\mathrm{L}-1} \sum_{j=S+1}^{\mathrm{L}-1} P_{i j}^{\mathrm{C}} \log _{2}^{\mathrm{P}_{\mathrm{i}}^{\mathrm{C}}}
$$

Hence, the total second-order local entropy of the object and the background can be written as

$$
\mathbf{H}_{\mathrm{T}}^{(2)}(\mathrm{S})=\mathbf{H}_{\mathrm{A}}^{(2)}(\mathrm{S})+\mathbf{H}_{\mathrm{C}}^{(2)}(\mathrm{S}
$$

The gray level corresponding to the maximum of $\mathrm{H}_{\mathrm{T}}^{(2)}(\mathrm{S})$ gives the optimal threshold for object background classification. Fig 6 shows the result after local entropy thresholding of MFR image.

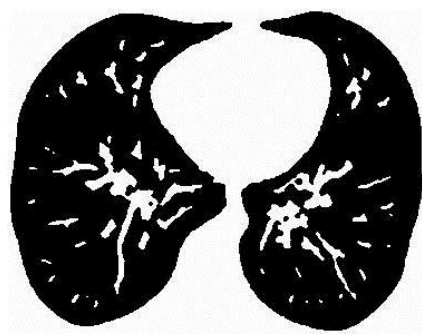

Fig 6: Local entropy thresholding result for the image shown in Fig 4 (MFR).

\section{RESULTS \& PERFORMANCE EVALUATION}

The proposed method is tested on publicly available databases of Lung TIME [16] which is very useful to extract pulmonary vessel tree form low dose chest CT scans obtained from the Motol Environment. For this purpose, a pilot clinical sample was also acquired to examine the exactness of the outcome. The results are compared to those obtained by Hessian-based filters output and they demonstrate that the proposed method exhibits better connected segmentations and is capable of finding connections to thin, peripheral vessels. Experiments were performed on 10 out of 157 low dose chest CT scans for which the pulmonary vessels are extracted.

In this section, experiments have been performed with both synthetic images and real chest CT images to verify the performance of the proposed matched filter in comparison with the filters introduced by Frangi and Shikata, which are considered as the standard techniques.

\subsection{Junction suppression}

A synthetic image of size 512 × 512 which was processed by the three filter models. The synthetic image is obtained to contain vessels of different sizes and junctions of different types. It is possible to see that the Frangi and Shikata filters suppress junctions while the matched filter approach does not. The suppressed junctions make vessels discontinuous. Although this error may be small, it can cause the splitting of a single vessel, which in turn has a critical effect on the vessel-tree reconstruction accuracy. Fig 7 shows the results of synthetic image.

Vessel has one principal direction, which is mathematically indicated by a small ratio between the minimum and maximum Hessian eigenvalue. Meanwhile, at a junction, where a vessel branches off, there are more than two principal directions, and thus the ratio of two eigenvalues is no longer small. As a result, the Frangi and Shikata filters considered these points as noise and hence they are suppressed. In the proposed method convolves the input image with twelve rotated versions of a two-dimensional matched filter $\left(15^{0}\right.$ of angular resolution). As the maximum response of the filter with respect to every angle is retained junctions are explored clearly.

\subsection{Noise sensitivity}

To compare the performances of the filters with respect to noise levels, we considered a set of phantom images shown in Fig 8. The properties of phantom are as follows. First of all, one original phantom image with various typical hindrances 
images for accurate vessel detection is created and later on used as the "ground truth". In this 512 x 512 sized phantom, fifteen vessel segments are constructed for a wide range of widths and directions to model as vascular image. For the sake of further analysis, these segments are numbered in an increasing order from left to right and top to bottom.

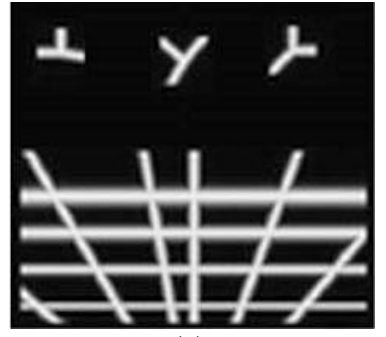

(a)

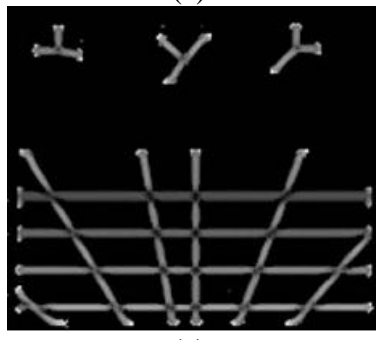

(c)

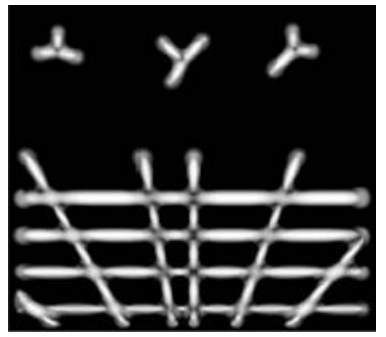

(b)

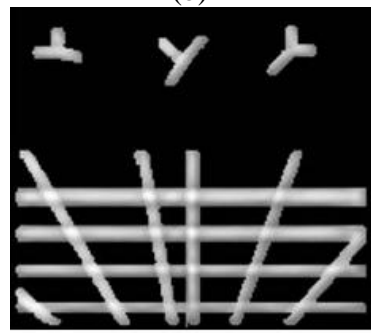

(d)
Fig 7: Vessel improvement results. (a) The original synthetic image. (b) enhanced image by the Frangi method. (C) by the Shikata method, and (d) by our approach. The Frangi and Shikata methods suppress the junctions while ours does not.

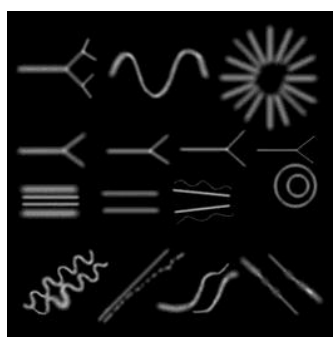

Fig 8: Test data with a size of $512 \times 512$ phantom obtained from www.ecse.rpi.edu/censsis/phantom/fp_d1108_2.pgm. The testing data incorporate most of the common challenges to exact vessel extraction procedure such as image noise and presence of close parallel vessels, very thin vessels, discontinued vessels, and vessels with variable intensities along their length.

Segment 1 represents different branch points in a real chest CT image. Segment 3 stands for vessel orientation diversity. Segments 4 to 7 characterize junctions with different widths. Moreover, segments 2, 12, and 14 are designed deliberately to have variable cross-sectional widths. Further, common challenges such as the presence of close parallel vessels in segments 8, 9, and 11, very thin vessels in segment 10 , discontinued vessels in segment 13 , and vessels with variable intensities along their length in segment 15 are also incorporated into the phantom. In the next step based on this original phantom, a series of testing data are generated by adding various levels of white noise, having standard deviation (SD) ranging from $5 \%$ to $80 \%$. The noise SD is calculated as a percentage of the 8-bit dynamic range of the image (0-255). To our experience, the data with noise SD of $80 \%$ represents the most possibly challenging situation, which is well beyond any worst case of real CT images.
Then the three filters are applied on those phantom images and the outputs are segmented using local entropy thresholding to compare with the "ground truth". The quantitative performance is measured with receiver operating characteristic (ROC) curves [17]. An ROC curve plots the rate of pixels correctly classified as vessels (i.e., true positive rate or sensitivity) against the rate of pixels incorrectly classified as vessels. The rates are obtained with all possible threshold choices. Each discrete threshold value produces a pair corresponding to a single point in the curve. The closer the curve approaches the northwest corner, the better the filter performs. A single scalar value reflecting this behavior is the area under the ROC curve (AUC), which is 1 for perfect performance. Note that to get rid of the large number of background pixels correctly classified can compute the sensitivity and specificity in the vicinity of the "ground truth" vessels which can be obtained by dilation. In our experiment, the vicinity size is selected such that the number of background pixels is comparable to that of the vessel pixels.

Fig 9 shows sample enhancement results for the data with noise SD of $20 \%$. The performances of the three filters applied on the whole testing data set are presented in Table 1 and Fig 10. In this figure, the AUC measures are plotted as a function of the noise SD. We can see that the matched filter out performs the others for this data set. Specifically, compared to the Frangi filter, it generates similar results in case of low noise (i.e., SD of $5-10 \%$ ) but performs much better when the noise level increases.

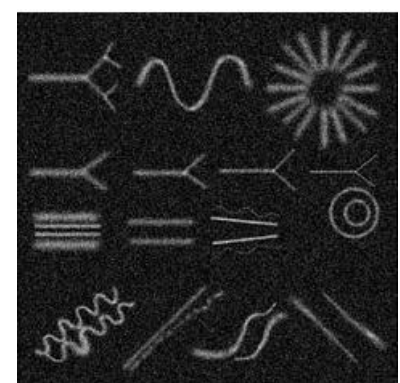

(a)

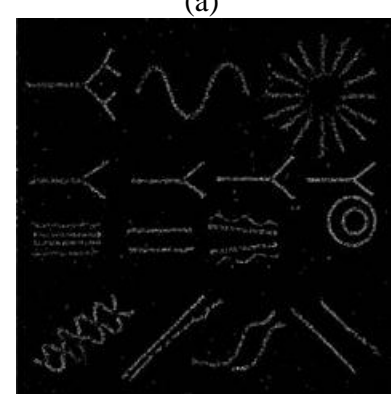

(c)

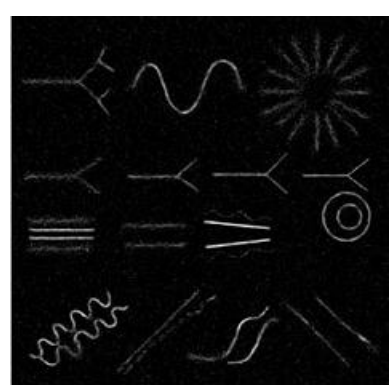

(b)

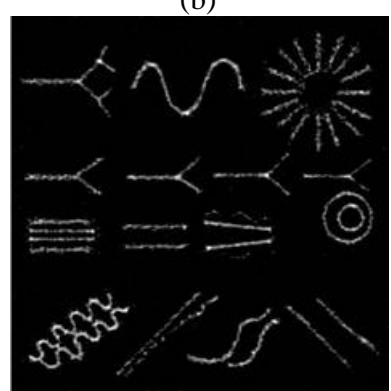

(d)
Fig 9: Sample vessel enhancement results. (a) Sample phantom image with noise SD of $20 \%$. (b) Enhanced image by the Frangi filter, CPU $=\mathbf{2 . 4 2} \mathrm{s}$, (c) by the Shikata filter, $2.24 \mathrm{~s}$, and (d) by Matched filter, $5.27 \mathrm{~s}$.

\subsection{Real data}

Similar to junction suppression problem, small vessel enhancement is critical because those thin vessels which may appear broken or disconnected from larger structures will often be omitted in the reconstruction procedures.

Fig 11 shows the enhancement results of the three filters applied on two real lung CT images. The images are of size $512 \times 512$ and belong to chest part of the human body. As can be observed, the Frangi filter gives good results with large 
vessels but fails to detect small ones while the Shikata model is able to enhance small vessels but unfortunately enhances background noise also. Conversely, the matched filter can enhance small vessels with more continuous appearances.

Table1: SD of the AUC of the three methods performed on the Fig. 9

\begin{tabular}{|c|c|c|c|}
\hline \multirow{2}{*}{ SD } & \multicolumn{3}{|c|}{ AUC } \\
\cline { 2 - 4 } & Frangi & Shikata & $\begin{array}{c}\text { Proposed } \\
\text { Matched }\end{array}$ \\
\hline 0 & 0.94 & 0.94 & 0.94 \\
\hline 10 & 0.94 & 0.91 & 0.94 \\
\hline 20 & 0.89 & 0.85 & 0.91 \\
\hline 30 & 0.88 & 0.84 & 0.89 \\
\hline 40 & 0.86 & 0.82 & 0.88 \\
\hline 50 & 0.82 & 0.80 & 0.84 \\
\hline 60 & 0.82 & 0.79 & 0.83 \\
\hline 70 & 0.77 & 0.77 & 0.80 \\
\hline 80 & 0.79 & 0.78 & 0.79 \\
\hline
\end{tabular}

The segmentation accuracy of the proposed method was evaluated quantitatively on a data set of 10 out of 157 patient scans by comparing automatically derived vessel segments with expert radiologist randomly selected 100 points from

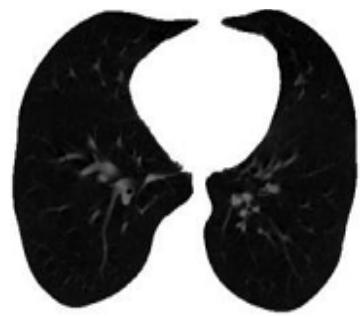

(a)

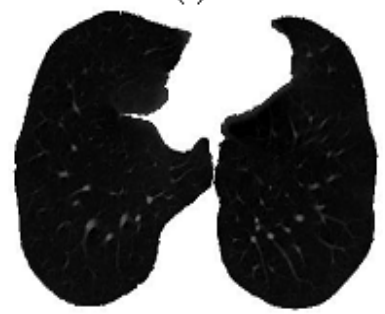

(e)

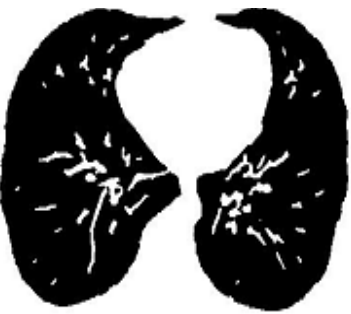

(b)

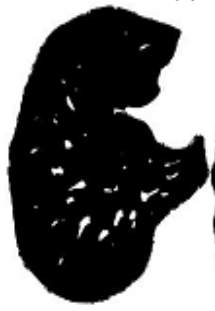

(f)

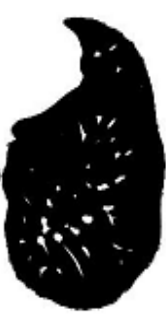

pulmonary vessels and 100 points in background near vessel structures for axial slices of CT images. Then matched filter and local entropy threshold are applied to these images and the above regions are used as the ground truth to calculate the sensitivity and specificity. Our final results show that sensitivity rate of $96.25 \%$ and specificity rate of $98.5 \%$ for our datasets.

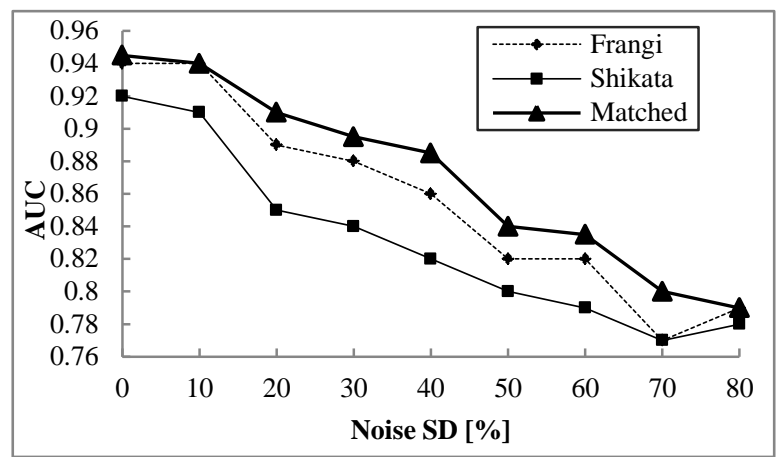

Fig 10: Performance plots vs. noise levels for the testing data described in Fig. 9. In average, the AUC of matched directional filter is relatively $13 \%$ and $36 \%$ larger than that of Frangi and Shikata filters, respectively.

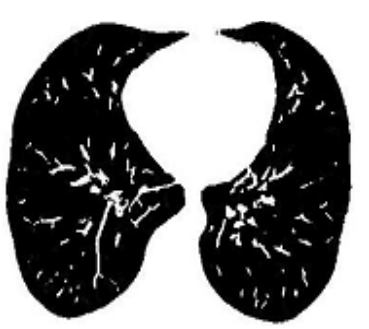

(c)

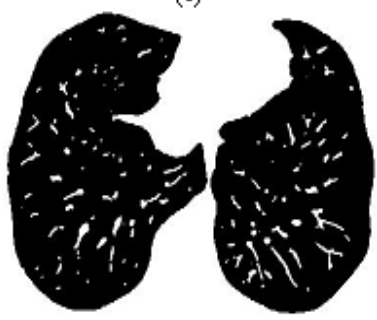

(g)

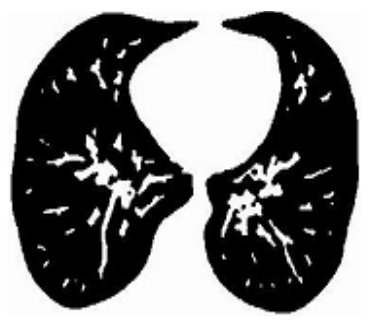

(d)

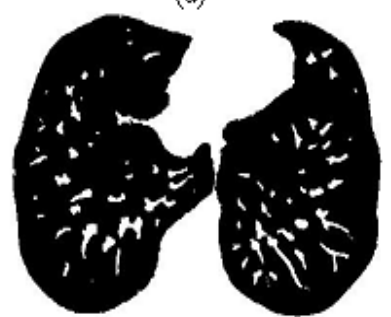

(h)

Fig 11: Qualitative results for two lung CT images. (a and e) Original images, (b and f) enhanced images by Frangi method, $\mathrm{CPU}=\mathbf{2 . 4 3} \mathrm{s}$ each, $(\mathrm{c}$ and $\mathrm{g})$ by Shikata method, $2.21 \mathrm{~s}$, and (d and $\mathrm{h}$ ) by Matched filter approach, $4.38 \mathrm{~s}$. The Frangi and Shikata models fail to correctly enhance small and thick vessels but our approach succeeds.

\section{DISCUSSIONS AND CONCLUSION}

In this paper we have presented a novel approach of vessel enhancement for 2D Chest CT images by using matched filter and local entropy thresholding. Our main contribution resides in adapting the matched filter kernels at different angles to be used in the lung region. In particular, this permits the estimation of the vessel directions without the Hessian eigenanalysis. The advantage of the proposed approach is that it distinguishes all vessels at bifurcations and crossings.

Hessian-based filters can enhance vessels of various sizes and simultaneously estimate their directions. However, Hessianbased filters cannot distinguish step edges from vessels effectively. Matched filters are capable of distinguishing step edges from vessels more effectively.
We believe the ratio of eigenvalues used in Frangi filter cannot distinguish edges from vessels as effectively as matched filters due to the fact that it only uses the local information, while the matched filter uses all pixels in the cross section of a vessel. Therefore, an additional filter response is obtained by convolving the image with a matched filter in the cross direction of the vessels. The matched filter is a second order derivative of one dimensional Gaussian function.

The experimental results show that the matched filter kernels at different angles overcome the limitations of conventional Hessian-based methods such as junction suppression and noise sensitivity. It also performs better on real chest CT images.

In conclusion, we consider the proposed matched filter and local entropy thresholding methods performs very well to 
extract thin lung blood vessels even in low contrast regions in clinical tasks. However, there is still room for improvement for detecting the finer vessels. Another difficulty of the proposed method is that in some images fissure of the lung region is miss-detected as blood vessel. The future work aims to solve these challenges.

\section{REFERENCES}

[1] E Li, S. Sone, H. Abe, H. MacMahon, S. Aimato Ill, and K. Doi. "Lung cancers missed at low-dose helical CT screening in a general population: comparison of clinical, histopathologic, and imaging findings," Radiology, vol. 225, pp. 673-683, 2002

[2] W. Huang, R. Yen, M. McLaurine, and G. Bledsoe, "Morphometry of the human pulmonary vasculature," Journal of Applied Physiology, vol. 81, pp. 2123-2133, 1996.

[3] Hidenoris hikata,Geoffrey McLennan, Eric A.Hoffman, and Milan Sonka, "Segmentation of Pulmonary Vascular Trees from Thoracic 3D CT Images", in Hindawi Publishing Corporation International Journal of Biomedical Imaging Volume 2009, Article ID636240, 11 pages doi:10.1155/2009/636240.

[4] O. Agam, S.O. Armato III. and C. Vu, "Vessel tree reconstruction in thoracic CT scans with application to nodule detection," IEEE Trans. Med. Imaging, vol. 24, no. 4, pp. 486-499, 2005.

[5] A. F. Frangi, W. J. Niessen, K. L. Vincken, and M. A. Viergever, "Multiscale vessel enhancement filtering," in Medical Inage computing and Computer Assisted Intervention, 1998, pp. 130-137.

[6] Panayiotis D. Korfiatis, Cristina Kalogeropoulou, Anna N. Karahaliou, Alexandra D. Kazantzi, and Lena I. Costaridou, "Vessel Tree Segmentationin Presence of Interstitial Lung Disease in MDCT", in IEEE Transactionson Information Technology in Biomedicine, vol.15, no.2, pp. 214-220, March 2011.

[7] Y. Sato, S. Nakajima, N. Shigara, H. Atsumi, T. Koller, G. Gerig, and R. Kikinis, "Three-dimensional multi-scale line filter for segmentation and visualization of curvilinear structures in medical images," Med.Img.Analysis, vol. 2, no. 2, pp. 143-168, 1998.

[8] Q. Li, S. Sone, arid K. Doi, "Selective enhancement filters for nodules, vessels, and airway walls in two- and three-dimensional CT scans," Medical Physics, vol. 30, no. 8, pp. 2040-2051, 2003.
[9] L. Cohcn and T. Deschamps, "Segmentation of 3D tubular objects with adaptive front propagation and minimal tree extraction for 3D medical imaging," Computational Methods in Biomechanics and Biomedical Engineering, vol. 10, no. 4, pp. 289-305, 2007.

[10] G. Song, A. Ramirez-Manzanares, and J. Gee, "A simultaneous segmentation and regularization framework for vessel extraction in CT images," in First international workshop on pulmonary image processing, pp. 185-193, 2008.

[11] Panayiotis D. Korfiatis, Anna N. Karahaliou, Alexandra D. Kazantzi, Cristina Kalogeropoulou, and Lena I. Costaridou, "Texture Based Identification and Characterization of Interstitial Pneumonia Patterns in Lung Multidetector CT" in IEEE Transactionson Information Technology in Biomedicine, vol.14, no.3, pp. 675-680, May 2010.

[12] Yanfeng Shang, Rudi Deklerck, Edgard Nyssen, Aneta Markova, Johande Mey, Xin Yang, and Kun Sun, "Vascular Active Contourfor Vessel Tree Segmentation", IEEE Transactions on Biomedical Engineering, vol.58,no.4, pp. 1023-1032, April 2011.

[13] N. Otsu, "A threshold selection method from gray-level histograms," IEEE Transactions on Systems,Man, and Cybernetics, vol. SMC-9, pp. 62-66, Jan. 1979.

[14] S. Chaudhuri, S. Chatterjee, N. Katz, M. Nelson, and M. Goldbaum, "Detection of blood vessels in retinal images using two dimensional matched filters," IEEE Trans. Medical imaging, vol. 8, no. 3,pp. 263-269, September 1989.

[15] Chang, C.-I.; Du, Y.; Wang, J.; Guo, S.-M.; Thouin, P.D., Survey and comparative analysis of entropy and relative entropy thresholding techniques, Vision, Image and Signal Processing, Page(s):837 - 850, IEE Proceedings, Vol. 153, 2006.

[16] M. Dolejsi, J. Kybic, M. Polovincak, and S. Tuma, "The Lung TIME-Annotated lung nodule dataset and nodule detection framework," in Proceedings of SPIE, Vol. 7260, 72601U, 2009.

[17] T. Fawcett, ROC Graphs: Notes and Practical Considerations for Researchers, Technical Report, HP Laboratories, Palo Alto, USA, 2004. 